\title{
INSULIN RESISTANCE IN END STAGE RENAL DISEASE (ESRD) PATIENTS IN EASTERN INDIA: A POPULATION BASED OBSERVATIONAL STUDY
}

\author{
Roy Himansu', *Banerjee Prithwijit ${ }^{2}$, Dan Subhasish ${ }^{3}$, Rahaman Musfikur ${ }^{4}$, Sengupta Mohua $^{5}$, Bal Chiranjeet $^{5}$ \\ 1. MS, Associate Professor, Department of General Surgery, Medical College Kolkata.
}

2. MD, Demonstrator, Department of Pharmacology, Medical College Kolkata.

3. MD, Demonstrator, Department of Biochemistry, Medical College Kolkata.

4. MD, Assistant Professor, Department of Pharmacology, Medical College Kolkata.

5. MD, Assistant Professor, Department of Pharmacology, Medical College Kolkata.

6. MD, Assistant Professor, Department of Physiology, Medical College Kolkata

*Corresponding Author's e-mail: drprithwijit@gmail.com, Contactno: +91-9830500513

\begin{abstract}
Background \& Objective: Despite considerable advances made in the field of medicine in general, and nephrology in particular, Chronic Kidney Disease (CKD) and End Stage Renal Disease (ESRD) remain public health challenges of enormous proportion. Aim: Considering that the association between Insulin resistance and CKD and ESRD has been established in studies conducted abroad, we propose to verify the association in Indian setting i.e. among ESRD patients in eastern India. Materials and Methods: 95 patients were included in control group and 97 patients were included in ESRD group. Only consenting individuals chosen through the filters of appropriate inclusion and exclusion criteria were made part of the study. Results: The statistical software Minitab 16 was used to analyze the data. Serum fasting Insulin level, HOMA-IR, serum hsCRP, serum urea, serum creatinine levels were significantly higher in ESRD patients compared to age and sex matched control individuals. Conclusion: Our study confirms the association between ESRD and IR found in other studies. Although the association between ESRD and IR has been established, the causality relation between them is not very clear. Further exploration of the issue is warranted.
\end{abstract}

Keywords: End Stage Renal Disease, Chronic Kidney Disease, Insulin Resistance

\section{INTRODUCTION:}

Despite considerable advances made in the field of medicine in general, and nephrology in particular, Chronic Kidney Disease (CKD) and End Stage Renal Disease (ESRD) remains a public health challenge of enormous proportion ${ }^{1}$. Studies have found that cardiovascular diseases are a leading cause of mortality for ESRD patients ${ }^{2}$. Cardiac mortality in dialysis patients is more than 10-fold greater than that of the general population $^{2}$. Of late, Insulin Resistance (IR) has been implicated as the underlying cause of a constellation of cardiovascular symptoms ${ }^{3}$. IR is common in patients with mild-to-moderate $\mathrm{CKD}^{4}$. Insulin resistance is also an independent predictor of cardiovascular mortality in End Stage Renal Disease ${ }^{5}$ (ESRD) and is increasingly recognized as a nontraditional risk factor ${ }^{6,7}$.

Considering that the association between Insulin resistance and CKD and ESRD has been established in studies conducted abroad, we propose to verify the association in Indian setting i.e. among ESRD patients in eastern India.

\section{MATERIALS AND METHODS:}

Patients were recruited from outpatient department of a reputed healthcare delivery centre in eastern India. This study was performed in accordance with the relevant ethical guidelines. Only patients/subjects providing written consent after being explained the study protocol were included in the study. 95 patients were included in control group and 97 patients were included in ESRD group. The following selection criteria were used for patient recruitment:

\section{Inclusion criteria:}

ESRD patients of either sex and their age and sex matched controls, who attended the OPDs during the study period and gave their voluntary written informed consent for the study.

\section{Exclusion Criteria:}

1. Obese patients

2. Diabetic patient

3. Metabolic syndrome patients

4. Endocrinopathy patients

5. Nephrotic syndrome

6. Pregnant and lactating mothers

7. Patients suffering from malignancy and terminally ill patients

8. Patients who are on drugs, known to cause Insulin resistance (e.g. Corticosteroids, Olanzapine etc) 
Normal renal function was defined according to the National Kidney Foundation Kidney Disease Outcome Quality Initiative (K/DOQI) definitions [estimated glomerular filtration rate (GFR) $\geq 120 \mathrm{ml} / \mathrm{min}$ per $1.73 \mathrm{~m}^{2}$ ]. Patients with GFR less than $15 \mathrm{ml} / \mathrm{min}$ per $1.73 \mathrm{~m}^{2}$ were included in ESRD group. GFR was calculated using MDRD formula.

None of the ESRD patients was on hemodialysis or peritoneal dialysis.

\section{Study Protocol:}

Before collection of data or blood sample, each patient was explained the details of the study including rationale, expected benefits, risk profile, confidentiality safeguards and study protocol. For some patients, help of appropriate interpreter(s) was taken. Only those patients who were willing to follow the study protocol and gave their written consent were included in the study. There was neither any financial cost nor any financial incentive for the patient for being part of the study.

1. Appropriate blood samples were collected from ESRD patients. For estimation of serum Insulin (Ins) level, serum high sensitive CRP (hsCRP) level, serum Cholesterol (Cho) level, serum Triglyceride (Tgl), serum High Density Lipoprotein (HDL) level, serum Low Density Lipoprotein (LDL) level, fasting blood sample was drawn into serum (without anticoagulant) gel containing yellow colour capped BD Vacutainer tubes. For plasma Glucose (Glu) level, blood sample were drawn into fluoride-oxalate anticoagulant containing gray colour capped BD Vacutainer tubes. All samples were immediately centrifuged, separated and stored at $2-8^{\circ} \mathrm{C}$ until analysis for the relevant biochemical parameters.

2. Plasma Glucose was measured by Dimension RXL Max autoanalyzer using Hexokinase principle.

3. Serum hsCRP was measured by Dimension RXL Max autoanalyzer instrument using immunoturbidimetric principle.

4. Serum Urea was measured by Dimension RXL Max autoanalyzer instrument using Urease principle.

5. Serum Creatinine was measured by Dimension RXL Max autoanalyzer instrument using Alkaline Picrate Kinetic principle.

6. Serum Triglyceride was measured by Dimension RXL Max autoanalyzer instrument using GPOPOD-AAP principle.

7. Serum Cholesterol was measured by Dimension RXL Max autoanalyzer instrument using CECHOD-AAP principle.
8. Serum HDL was measured by Dimension RXL Max autoanalyzer instrument using AHDL method.

9. Serum LDL was measured by Dimension RXL Max autoanalyzer instrument using ALDL method.

10. Serum Ins was measured by Abbott AxSYM is based on Microparticle Enzymes Immunoassay (MEIA) technique.

11. Serum albumin was measured by Dimension RXL Max autoanalyzer instrument using bromocresol purple (BCP) dye-binding method.

12. Patients' recent-most blood/plasma/serum values of the afore-mentioned biochemical parameters were noted if already available provided they were done on the same day.

13. Patients' current medication details were noted.

14. Patients' demographic profile including age, sex was noted.

Post data collection; patients were divided into two groups - CKD and Control. The serum levels of Ins, Cho, Tgl, HDL, LDL and plasma Glu between the two groups were compared for presence or absence of statistical significance.

Homeostatic Model Assessment of Insulin Resistance (HOMA-IR) was calculated by the formula:

HOMA-IR = Fasting Serum Insulin $(\mu \mathrm{IU} / \mathrm{mL}) \times$ Fasting Plasma Glucose (mg/dL) / 405

\section{Statistical Analysis:}

The statistical software Minitab 16 was used to analyze the data. Values of continuous and normally distributed variables were expressed as mean \pm standard deviation unless otherwise indicated, and differences in mean values between two groups were analysed using Student's t-test. Descriptive information regarding categorical variable were presented as frequency. Test for equality of two proportions was used for comparison of categorical data. All tests were two tailed and considered statistically significant if $\mathrm{p}$-value is $<0.05$.

\section{RESULTS:}

Serum fasting Insulin level, HOMA-IR, serum hsCRP, serum urea, serum creatinine levels were significantly higher in ESRD patients compared to age and sex matched control individuals. Serum albumin level was significantly lower in ESRD patients compared to age and sex matched control individuals. (Refer Table 1) 
Table 1: Comparison of anthropological, demographic and biochemical statistics between CKD and control groups

\begin{tabular}{|l|l|l|l|}
\hline \multicolumn{1}{|c|}{ Control } & \multicolumn{1}{c|}{ ESRD } & \multicolumn{1}{c|}{ p-value } \\
\hline Sample size & 95 & 97 & \multicolumn{1}{c|}{} \\
\hline Male/Female & $50 / 45$ & $51 / 46$ & 0.994 \\
\hline Age & $45.6 \pm 9.2$ & $47.3 \pm 8.1$ & 0.175 \\
\hline BMI & $25.2 \pm 5.3$ & $23.9 \pm 7.2$ & 0.155 \\
\hline Fasting Plasma Glucose $(\mathrm{mg} / \mathrm{dL})$ & $98 \pm 22$ & $103 \pm 25$ & 0.142 \\
\hline Fasting Serum Insulin $(\mu \mathrm{IU} / \mathrm{mL})$ & $8.2 \pm 2.6$ & $15.3 \pm 2.5$ & $<0.001^{*}$ \\
\hline HOMA-IR & $1.98 \pm 0.14$ & $3.89 \pm 0.15$ & $<0.001^{*}$ \\
\hline hsCRP $(\mathrm{mg} / \mathrm{L})$ & $3.6 \pm 1.8$ & $7.6 \pm 3.4$ & $<0.001^{*}$ \\
\hline Triglyceride $(\mathrm{mg} / \mathrm{dL})$ & $148 \pm 45$ & $158 \pm 59$ & 0.188 \\
\hline Total Cholesterol $(\mathrm{mg} / \mathrm{dL})$ & $176 \pm 49$ & $189 \pm 45$ & 0.057 \\
\hline LDL $(\mathrm{mg} / \mathrm{dL})$ & $87 \pm 22$ & $92 \pm 26$ & 0.152 \\
\hline HDL $(\mathrm{mg} / \mathrm{dL})$ & $42 \pm 10.2$ & $39 \pm 9.8$ & $0.039^{*}$ \\
\hline Urea $(\mathrm{mg} / \mathrm{dL})$ & $29 \pm 9$ & $69 \pm 18$ & $<0.001^{*}$ \\
\hline Creatinine $(\mathrm{mg} / \mathrm{dL})$ & $0.9 \pm 0.4$ & $5.9 \pm 1.1$ & $<0.001^{*}$ \\
\hline Serum Albumin $(\mathrm{g} / \mathrm{dL})$ & $4.5 \pm 1.0$ & $4.0 \pm 1.2$ & $0.002^{*}$ \\
\hline
\end{tabular}

\section{DISCUSSION:}

Our study confirms the association between ESRD and IR found in other studies ${ }^{8,9,10}$.

Although the association between ESRD and IR has been established, the causality relation between them is not very clear. It is not yet clear whether ESRD causes IR in non-diabetic, non-hypertensive patients or IR leads to ESRD. Evidences supporting either direction of the causality relation have been found.

IR has been known to be present even in early stages of CKD. Glucose Disposal Rate (GDR) in hyperinsulinemic euglycemic clamp test has been found to correlate negatively with plasma creatinine and positively with creatinine clearance rate ${ }^{4}$. Data from the Atherosclerosis Risk in Communities (ARICs) cohort confirmed a stepwise increase in risk for ESRD development with the number of MetS criteria met in nondiabetic adults, ever after controlling for the development of diabetes mellitus (DM) and hypertension ${ }^{11}$. However, there is little evidence that preventing or treating symptoms of the MetS protects patients with renal impairment.

IR, along with oxidative stress and inflammation, is suggested to play a role in the development of albuminuria and declining kidney function ${ }^{12,}{ }^{13}$. IR promotes kidney disease by worsening renal hemodynamics through mechanisms such as activation of the sympathetic nervous system ${ }^{14}$, sodium retention, decreased $\mathrm{Na}+\mathrm{K}+-\mathrm{ATPase}$ activity, and increased $\mathrm{GFR}^{13}$. Endoplasmic reticulum (ER) stress seems to be the factor-linking inflammation and IR at the molecular level. Renal ER stress is associated with proteinuriainduced podocyte damage and alteration of nephrin $\mathrm{N}$ glycosylation in podocytes, which is the underlying factor in the pathogenesis of proteinuria, and is involved in the pathophysiology of chronic kidney injury with tubulointerstitial damage $\mathrm{e}^{15}$. IR and inflammatory cytokine release may be partly responsible for glomerular mesangial expansion, basement membrane thickening, podocytopathy, and the loss of slit pore diaphragm integrity ultimately leading to glomerulosclerosis and tubulointerstitial injury ${ }^{16}$.

Like other chronic diseases, patient with ESRD demonstrates low-grade systemic inflammation marked by elevated levels of proinflammatory cytokines ${ }^{17}$ such as C-reactive protein (CRP), tumor necrosis factor alpha (TNF- $\alpha$ ), inerleukin-6 (IL-6), and interleukin-1 beta (IL$1 \beta)$. Inflammation and oxidative stress are evident in the early stage of $\mathrm{CKD}^{18}$ and are also known to induce IR, primarily via increased production of proinflammatory cytokines.

There are evidences that the vitamin $\mathrm{D}$ and or parathyroid hormone (PTH) axis is important in the pathogenesis of glucose intolerance and IR in patients with ESRD. It has been found that IR is present in the early stages of ESRD and has an inverse association with 25-hydroxyvitamin D levels ${ }^{19}$. The PTH level is usually elevated in ESRD patients. The mechanisms through which excess PTH blunts insulin sensitivity are uncertain.

\section{CONCLUSION:}

To the best of our knowledge, this is the first study in eastern India exploring the effect of ESRD on insulin resistance. Limitations of our study include a crosssectional design. In addition, results in this study may not be applicable to general population or to patients with hypertension in a primary care clinic because patients in an outpatient clinic in a hospital are selected patients.

Conflict of interest: There was nothing to the best of our knowledge. 


\section{REFERENCES}

1 Kasiske BL, Chavers B, Foley R et al. K/DOQI clinical practice guidelines for chronic kidney disease: evaluation, classification, and stratification. American Journal of Kidney Disease 2002; 39(supplement 1, no. 2):S1-S266.

2 Foley RN, Parfrey PS, and Sarnak MJ. Clinical epidemiology of cardiovascular disease in chronic renal disease. American Journal of Kidney Diseases 1998; 32(5):S112-S119.

3 Frank B. Hu and Meir J. Stampfer et al. Insulin Resistance and Hypertension: The Chicken-Egg Question Revisited. Circulation 2005; 112:1678-1680.

4 Kobayashi S, Maesato K, Moriya H, Ohtake T, Ikeda T. Insulin resistance in patients with chronic kidney disease. American Journal of Kidney Diseases 2005; 45(2):275-280.

5 Shinohara K, Shoji T, Emoto M et al. Insulin resistance as an independent predictor of cardiovascular mortality in patients with end-stage renal disease. Journal of the American Society of Nephrology 2002; 13(7):1894-1900.

6 Becker BN, Himmelfarb J, Henrich WL, Hakim RM. Reassessing the cardiac risk profile in chronic hemodialysis patients: a hypothesis on the role of oxidant stress and other non-traditional cardiac risk factors. Journal of the American Society of Nephrology 1997; 8(3):475486.

7 Cheung AK, Sarnak MJ, Yan G et al. Atherosclerotic cardiovascular disease risks in chronic hemodialysis patients. Kidney International 2000; 58(1):353-362.

8 Hager SR. Insulin resistance of uremia. Am J Kidney Dis 1989; 14:272-276.

9 Fliser D, Pacini G, Engelleiter R et al. Insulin resistance and hyperinsulinemia are already present in patients with incipient renal disease. Kidney Int 1998; 53:1343-1347.

10 Seoung Woo Lee et al. Insulin resistance and muscle wasting in non-diabetic end- stage renal disease patients. Nephrol Dial Transplant $2007 ; 22: 2554-2562$.

11 Kurella M, Lo JC, Chertow GM, "Metabolic syndrome and the risk for chronic kidney disease among nondiabetic adults," Journal of the American Society of Nephrology 2005; 16(7):2134-2140.

12 Cheng HT, Huang JW, Chiang CK, Yen CJ, Hung KY, and K.D. Wu. Metabolic syndrome and insulin resistance as risk factors for development of chronic kidney disease and rapid decline in renal function in elderly. Journal of Clinical Endocrinology and Metabolism 2012; 97(4):1268-1276.

13 Gluba A, Mikhailidis DP, G. Y. Lip et al. Metabolic syndrome and renal disease. International Journal of Cardiology. In press.

14 Rowe JW, Young JB, and K. L. Minaker, "Effect of insulin and glucose infusions on sympathetic nervous system activity in normal man," Diabetes 1981;30(3):219-225.

15 Cybulsky AV. Endoplasmic reticulum stressin proteinuric kidney disease. Kidney International 2010; 77(3):187-193.

16 Sowers JR. Metabolic risk factors and renal disease. Kidney International 2007; 71(8): 719-720,.

17 Ikizler TA. Nutrition, inflammation and chronic kidney disease. Current Opinion in Nephrology and Hypertension 2008; 17(2):162-167.

18 Menon V, Rudym D, Chandra P, D. Miskulin, R. Perrone, and M. Sarnak. Inflammation, oxidative stress, and insulin resistance in polycystic kidney disease. Clinical Journal of the American Society of Nephrology 2011; 6(1):7-13.

19 Chonchol M and Scragg R. 25-Hydroxyvitamin D, insulin resistance, and kidney function in the Third National Health and Nutrition Examination Survey. Kidney International 2007; 71(2): 134-139. 